Chinese Journal of Organic Chemistry

NOTE

\title{
伊马替尼衍生物的合成及细胞毒活性研究
}

\author{
陈仕杰 $a$ 和 龙 ${ }^{a, b}$ 王雪微 ${ }^{a}$ 龚显峰 ${ }^{a}$ 张 华*, $a, b$ \\ ( ${ }^{a}$ 黑龙江大学功能有机重点实验室 哈尔滨 150080) \\ $\left({ }^{b}\right.$ 国药一心制药有限公司 长春 130012)
}

\begin{abstract}
摘要 以 3-乙酰基嘧啶、2-甲基-5-硝基苯胺为起始原料, 经加成、缩合、环化、还原得到中间体 $N$-(2-甲基-5-氨基苯 基)-4-(3-吡啶基)嘧啶-2-胺(6), 再与( $L)-N$-酰化-氨基酸缩合得到 14 个伊马替尼氨基酸衍生物 7a $\sim 7 \mathbf{n}$. 目标化合物结构 经过 IR, ${ }^{1} \mathrm{H}$ NMR, ${ }^{13} \mathrm{C}$ NMR, HRMS 等确证. 采用四甲基偶氮唑盐(MTT)法考察了目标化合物对人白血病细胞(K562)、 人肺癌细胞(A549)、人肝癌细胞(HepG-2)体外抑制活性测试. 结果显示化合物 7a,7d, 7e, 7i, 7j, 7k, 7l, 7n 体外抑制活性 较高, 与对照品伊马替尼相近.
\end{abstract}

关键词 伊马替尼衍生物; 合成; 四甲基偶氮唑盐(MTT)法

\section{Synthesis and Cytotoxic Activity of Imatinib Derivatives}

\author{
Chen, Shijie ${ }^{a} \quad$ He, Long $^{b} \quad$ Wang, Xuewei $^{a} \quad$ Gong, Xianfeng ${ }^{a} \quad$ Zhang, Hua ${ }^{*, a, b}$ \\ ( ${ }^{a}$ Key Laboratory of Applied Organic, Heilongjiang University, Harbin 150080) \\ ( ${ }^{b}$ Sinopharm A-Think Pharmaceuticals Co., Ltd., Changchun 130012)
}

\begin{abstract}
Fourteen derivatives of imatinib have been prepared by condensation of $(L)$ - $N$-acylation amino acid with $N$-(5-amino-2-methylphenyl)-4-(3-pyridyl)-2-pyrimidine amine (6), which was prepared from 3-acetyl-pyrimidin and 2-methyl-5-nitroaniline through the reactions of addition, condensation, cyclization and reduction, respectively. The structures of all target compounds were characterized by IR, ${ }^{1} \mathrm{H}$ NMR, ${ }^{13} \mathrm{C}$ NMR and HRMS techniques. They were evaluated for cytotoxic activity against human Leukemia cells (K562), human non-small-cell-lung cancer cells lines (A549) and human hepatoma cell lines (HepG-2) by methyl thiazolyl tetrazolium (MTT) method. The results showed the cytotoxic activities of compounds $\mathbf{7 d}, \mathbf{7 i}, \mathbf{7 j}, \mathbf{7 k}, \mathbf{7 l}, \mathbf{7 n}$ against human Leukemia cells (K562) and human non-small-cell-lung cancer cell lines (A549), compounds $\mathbf{7 a}, \mathbf{7 d}, \mathbf{7 e}$ against human hepatoma cell lines (HepG-2) were comparable to those of imatinib.
\end{abstract}

Keywords imatinib derivatives; synthesis; methyl thiazolyl tetrazolium (MTT) assay

甲磺酸伊马替尼(Imatinib Mesilate, 图 1), 化学名 为 4-[(4-甲基-1-哌嗪)甲基]- $N$-[4-甲基-3-[[4-(3-吡啶)-2嘧啶]氨基]苯基]-苯胺甲磺酸盐, 由瑞士诺华(Novartis) 公司开发, 属于小分子蛋白酪氨酸激酶抑制剂, 临床上 用于治疗慢性髓细胞白血病 (chronic myelocytic leuke$\mathrm{mia}, \mathrm{CML})^{[1]}$, 其作用机制是针对酪氨酸激酶 bcr/ abl 亚 型受体，选择性结合于 $\mathrm{bcr} / \mathrm{abl}$ 蛋白酪氨酸激酶催化区的 核苷酸, 竞争性地阻止酪氨酸激酶与 ATP 结合, 降低酪 氨酸激酶活性, 从而抑制肿瘤细胞的增殖、转 移 ${ }^{[2,3]}$. 2001 年获得美国食品药品监督管理局(FDA)批准上市 ${ }^{[4,5]}$, 当年即取得了 1.65 亿美元的显赫销售佳绩, 短短三 年就培育成了 “重磅炸弹” 药物. 2004 年销售额已达
16.34 亿美元. 目前该药已在世界 90 多个国家和地区销 售, 2014 年销售额达 47.60 亿美元.<smiles>Cc1ccc(NC(=O)c2ccc(CN3CCN(C)CC3)cc2)cc1Nc1nccc(-c2cccnc2)n1</smiles>

图 1 甲磺酸伊马替尼

Figure 1 Imatinib mesylate

甲磺酸伊马替尼在慢性髓细胞白血病的治疗上取 得了显著的疗效，但是随着临床上的应用，其耐药性和

\footnotetext{
*E-mail: zhanghua34@163.com

Received June 25, 2015; revised July 27, 2015; published online August 26, 2015.

Project supported by the Foundation of Heilongjiang Educational Committee (No. 12521417)

黑龙江省教育厅科学技术研究(No. 12521417)资助项目.
} 
不良反应如水肿、恶心、呕吐、皮疹、骨关节及肌肉疼 痛、中性粒细胞及血小板减少等相继出现 ${ }^{[6 \sim 9]}$. 临床研 究报道该药在急性变期 CML 平均使用 10.5 周后疗效明 显下降. Sawyers 等 ${ }^{[10]}$ 曾报道 291 例 CML 患者在平均接 受伊马替尼治疗 $123 \mathrm{~d}$ 后, 伊马替尼的耐药率为 14.44\%(其中 21 例男性, 21 例女性). 因此合成伊马替尼 衍生物, 研究开发其 “Me-too”或 “Me-better” 药物具有 重要应用价值. 本工作尝试将 $L$-氨基酸引入伊马替胺母 体结构中, 希望能够增加化合物的溶解性, 增加药物分 子与酪氨酸激酶催化区域的特异性空间结合位点, 从而 竞争性地阻止酪氨酸激酶与 ATP 结合, 降低酪氨酸激 酶活性. 本工作参考文献报道 ${ }^{[11 ~ 13]}$, 以 3-乙酰基嘧啶、 2-甲基-5-硝基苯胺为起始原料, 经加成、缩合、环化、 还原得到关键中间体 $N$-(2-甲基-5-氨基苯基)-4-(3-吡啶 基) 嘧啶-2-胺 (6), 最后采用综合剂 $O$ - 苯并三唑$N, N, N^{\prime}, N^{\prime}$-四甲基脲四氟硼酸酯(TBTU)使中间体 $\mathbf{6}$ 与 $(L)-N$-酰化-氨基酸缩合得到 14 个光学活性的伊马替尼 氨基酸衍生物 7a $\sim 7 \mathbf{n}$ (Scheme 1). 并对这 14 个目标衍 生物进行了人白血病细胞(K562)、人肺癌细胞(A549)、

人肝癌细胞(HepG-2)体外抑制活性测试.

\section{1 结果与讨论}

\section{1 波谱解析}

以目标化合物 $7 \mathbf{a}$ 的光谱数据分析为例. 化合物 $7 \mathbf{a}$
的 ${ }^{1} \mathrm{H}$ NMR 中 $\delta 10.04$ 出现酰胺活泼氢, $\delta 7.15 \sim 9.27$ 出 现 14 个芳环氢, 高场 $\delta 1.80 \sim 2.20$ 出现两个甲基单峰, $\delta$ 2.85 出现两个氢峰, $\delta 4.67$ 出现一个氢峰, 分子结构中存 在四个脂肪碳 $\delta 18.1,22.9,38.3,55.4 ; 21$ 个芳环碳 $\delta$ $108.0,116.1,116.6,124.3,126.8,127.6,128.5,129.6$, $134.9,148.7,151.8,159.9,161.6,162.1$ (部分芳环碳化学 位移重合); 两个酰胺羰基碳 $\delta 169.7,170.6$. 其红外光谱 图中 $3450,3289 \mathrm{~cm}^{-1}$ 处有吸收峰说明分子中有酰胺氢 存在; $3029 \mathrm{~cm}^{-1}$ 为苯环 $=\mathrm{CH}$ 伸缩振动吸收峰, $2969 \mathrm{~cm}^{-1}$ 为甲基或亚甲基 $\mathrm{C}-\mathrm{H}$ 伸缩振动吸收峰, $1664 \mathrm{~cm}^{-1}$ 为酰 胺羰基伸缩振动吸收峰, $1645,1602,1578,1532 \mathrm{~cm}^{-1}$ 为 苯环 $\mathrm{C}=\mathrm{C}$ 伸缩振动吸收峰, 高分辨质谱 $\mathrm{HRMS}$ (ESI-MS) calcd for $\mathrm{C}_{27} \mathrm{H}_{27} \mathrm{~N}_{6} \mathrm{O}_{2}[\mathrm{M}+\mathrm{H}]^{+} 467.2190$, found 467.2190 , 说明分子量正确. 综合 ${ }^{1} \mathrm{H} N M R,{ }^{13} \mathrm{C} N M R$, IR, HRMS 等光谱数据, 可以充分说明其结构的正确性.

\section{2 生物活性测试结果与讨论}

采用 MTT 比色法考察 14 个目标化合物对体外培养 的人白血病细胞(K562)、人非小细胞肺癌细胞(A549)和 人肝癌细胞(HepG-2)生长的抑制作用, 结果发现 $\mathrm{R}^{2}$ 侧 链基团较小的化合物 7d, 7i, 7j, 7k, 7l, 7n 对人白血病细 胞(K562)和人非小细胞肺癌细胞(A549)体外生长抑制作 用较强, $\mathrm{R}^{1}$ 侧链基团较小的化合物 7a, $7 \mathbf{d}, 7 \mathrm{e}$ 对人肝癌 细胞(HepG-2)的体外生长抑制作用较强(表 2).

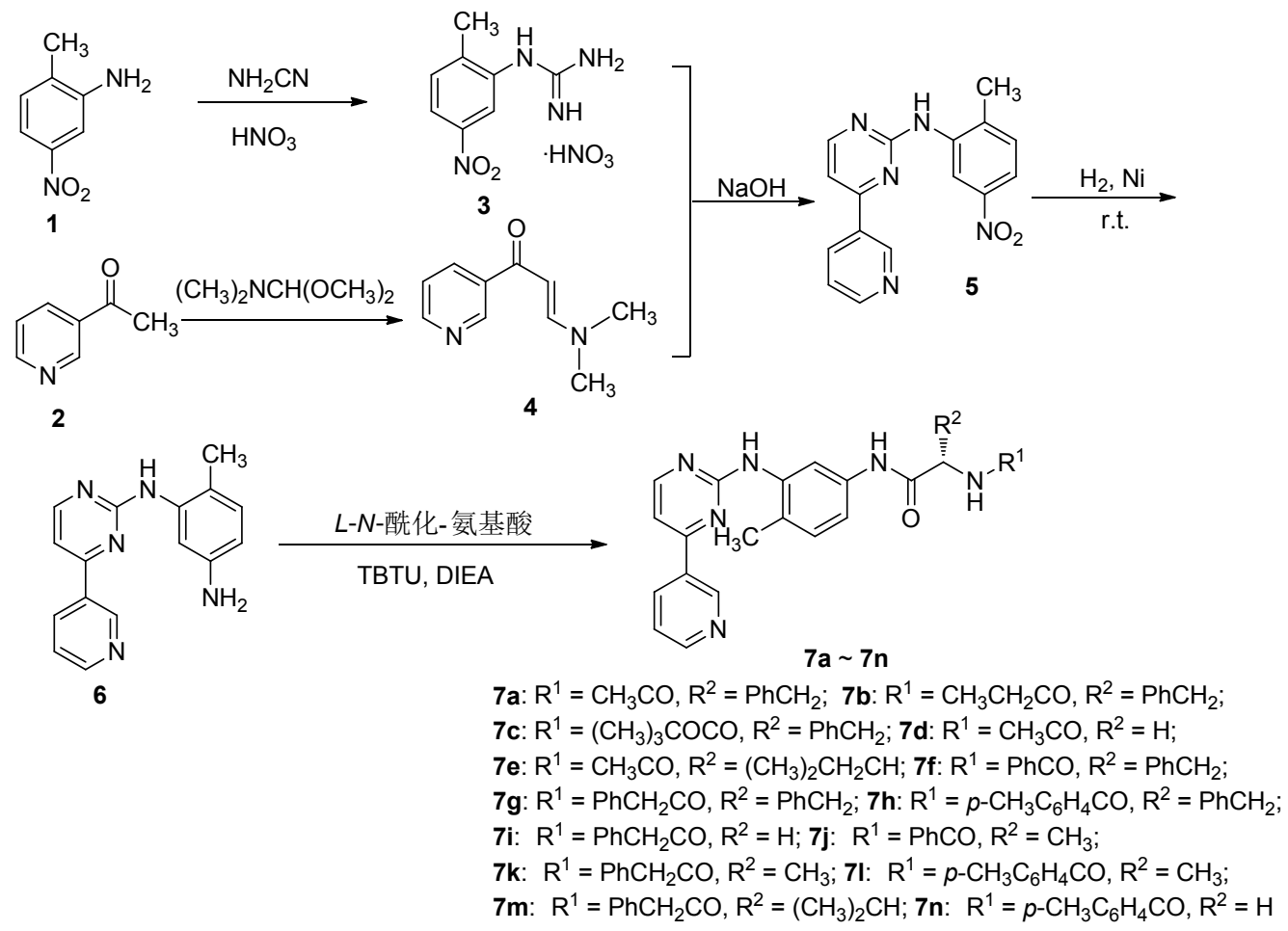

图式 1 化合物 7a 7n 合成路线

Scheme 1 Synthetic routes of target compounds $7 \mathbf{a} \sim 7 \mathbf{n}$ 
表 2 目标化合物体外对人 K562, A549 和 HepG-2 细胞的抑制 活性 $(c=150 \mu \mathrm{mol} / \mathrm{L})$

Table 2 Inhibitory activity of target compounds against K562, A549 and HEPG-2 cell growth in vitro $(c=150 \mu \mathrm{mol} / \mathrm{L})$

\begin{tabular}{cccc}
\hline \multirow{3}{*}{ Compound } & \multicolumn{3}{c}{ 抑制率/\% } \\
\cline { 2 - 4 } & K562 & A549 & HepG-2 \\
\hline $\mathbf{7 a}$ & 20.5 & 18.1 & 56.8 \\
$\mathbf{7 b}$ & 28.2 & 17.4 & 23.0 \\
$\mathbf{7 c}$ & 28.8 & 19.2 & 17.2 \\
$\mathbf{7 d}$ & 66.3 & 59.0 & 49.2 \\
$\mathbf{7 e}$ & 13.5 & 16.3 & 56.5 \\
$\mathbf{7 f}$ & 23.2 & 13.2 & 27.8 \\
$\mathbf{7 g}$ & 27.5 & 17.5 & 26.9 \\
$\mathbf{7 h}$ & 23.9 & 13.9 & 25.3 \\
$\mathbf{7 i}$ & 60.2 & 60.1 & 28.4 \\
$\mathbf{7 j}$ & 64.3 & 61.3 & 27.1 \\
$\mathbf{7 k}$ & 61.2 & 61.5 & 26.1 \\
$\mathbf{7 1}$ & 63.3 & 59.3 & 17.8 \\
$\mathbf{7 m}$ & 28.9 & 18.9 & 27.1 \\
$\mathbf{7 n}$ & 62.0 & 58.6 & 19.4 \\
Imatinib & 61.7 & 61.2 & 50.6 \\
\hline
\end{tabular}

\section{2 结论}

该类伊马替尼氨基酸衍生物 $7 \mathrm{a} \sim 7 \mathrm{n}$ 对人白血病细 胞(K562)、人非小细胞肺癌细胞(A549)和人肝癌细胞 (HepG-2)的生长均有一定程度的抑制作用. 其中化合物 $7 \mathbf{a}, 7 \mathrm{~d}, 7 \mathrm{e}, 7 \mathbf{i}, 7 \mathbf{j}, 7 \mathbf{k}, 7 \mathbf{l}, 7 \mathrm{n}$ 的抑制作用与对照品伊马替 尼的抑制活性相当或略高.

\section{3 实验部分}

\section{1 仪器与试剂}

红外光谱仪为 Spectrum One(溴化钾压片); 核磁共 振光谱仪为 Avance III400 (TMS 为内标, $\mathrm{CDCl}_{3}$ 或 DMSO- $d_{6}$ 为溶剂); 高分辨质谱仪为 micrOTOF-Q II10203; X-4 型数显显微熔点测定仪(温度计未校正); 各种 $(L)-N$-酰化氨基酸(纯度 $99 \%$, 上海瀚鸿化工科技有 限公司), 伊马替尼对照品(国药一心制药有限公司提 供), 其他化学试剂和原料均为化学纯或分析纯.

\section{2 实验方法}

\subsubsection{2-甲基-5-硝基苯胍硝酸盐(3)的合成}

将 2-甲基-5-硝基苯胺( $40 \mathrm{~g}, 0.26 \mathrm{~mol}$ )和单腈胺(22.0 $\mathrm{g}, 0.55 \mathrm{~mol}$ )溶于 $200 \mathrm{~mL}$ 异丙醇中, 摚拌, 加热到 $80{ }^{\circ} \mathrm{C}$, 于此温下缓慢滴入 $35 \mathrm{~mL}$ 浓盐酸, 滴毕, 维持此温摚拌 $1 \mathrm{~h}$, 然后再于 $80{ }^{\circ} \mathrm{C}$ 再缓慢滴入 $15 \mathrm{~mL}$ 浓盐酸, 并再维 持此温继续摚拌 $2 \mathrm{~h}$, 将反应混合物冷却到 $60{ }^{\circ} \mathrm{C}$, 逐滴 加入 $65 \%$ 的浓硝酸 $(20 \mathrm{~mL}, 0.28 \mathrm{~mol})$, 搅拌, 自然冷却 到室温, 析出白色固体, 过滤, 异丙醇洗涤, 干燥得产 物(60.0 g, 89\%), m.p. $222 \sim 224{ }^{\circ} \mathrm{C}$ (文献值 ${ }^{[14]}$ : m.p. $\left.219 \sim 226{ }^{\circ} \mathrm{C}\right)$.

\subsubsection{3-二甲氨基-1-(3-吡啶基)-2-丙烯-1-酮(4)的合成}

将 3-乙酰基吡啶(50 g, $0.42 \mathrm{~mol}$ )和 $N, N$-二甲基甲酰 胺二甲基缩醛(74.0 g, 0.64mol)混合溶于 $200 \mathrm{~mL}$ 二甲苯 中, 搅拌, 加热回流 $6 \mathrm{~h}$, 减压蒸干溶剂及 $N, N$-二甲基甲 酰胺二甲基缩醛等, 残留物用石油醚/丙酮 $(V: V=5$ :

1)重结晶得淡黄色固体( $66.0 \mathrm{~g}, 90 \%$ ), m.p. $65 \sim 67{ }^{\circ} \mathrm{C}$ (文 献值 ${ }^{[15]}$ : m.p. $66 \sim 67{ }^{\circ} \mathrm{C}$ ).

1.2.3 $\mathrm{N}$-(2-甲基-5-硝基苯基)-4-(3-吡啶基)嘧啶-2-胺 (5)的合成

将 3-二甲氨基-1-(3-吡啶基)-2-丙烯-1-酮(44.0 g, $0.25 \mathrm{~mol}$ )及 2-甲基-5-硝基苯胍硝酸盐 $(56.0 \mathrm{~g}, 0.22 \mathrm{~mol}$ ) 溶于 $150 \mathrm{~mL}$ DMF 中, 搅拌, 逐滴加入 $\mathrm{NaOH}$ 水溶液 $(8.8$ $\mathrm{g} \mathrm{NaOH}$ 溶于 $20 \mathrm{~mL}$ 水中), 滴毕, 加热到 $140 \sim 145{ }^{\circ} \mathrm{C}$ 反应 $8 \mathrm{~h}$, 冷却到 $80{ }^{\circ} \mathrm{C}$, 缓慢加入 $1 \mathrm{~L}$ 水, 搅拌, 自然冷 却到室温，过滤，甲醇重结晶得淡黄色粉末(54.0 g, $80 \%$ ). m.p. $196 \sim 198{ }^{\circ} \mathrm{C}$ (文献值 ${ }^{[15]}$ : $195 \sim 198{ }^{\circ} \mathrm{C}$ ).

1.2.4 N-(2-甲基-5-氨基苯基)-4-(3-吡啶基)嘧啶-2-胺 (6) 的合成

$\mathrm{N}$-(2-甲基-5-硝基苯基)-4-(3-吡啶基)嘧啶-2-胺(30.0 $\mathrm{g}, 0.098 \mathrm{~mol}$ )溶于 $1 \mathrm{~L}$ 四氢呋喃中, 加入 $15 \mathrm{~g}$ 新制的雷尼 镍, 常温常压催化氢化 $12 \mathrm{~h}$, 过滤, 减压浓缩, 乙醇重 结晶得到黄色结晶( $24.6 \mathrm{~g}, 90.3 \%$ ). m.p. 139 $140{ }^{\circ} \mathrm{C}$ (文 献值 ${ }^{[15]}$ : $\left.138 \sim 140{ }^{\circ} \mathrm{C}\right) ;{ }^{1} \mathrm{H}$ NMR $\left(400 \mathrm{MHz}, \mathrm{CDCl}_{3}\right) \delta$ : $9.27(\mathrm{~s}, 1 \mathrm{H}), 8.73 \sim 8.72(\mathrm{~d}, J=4 \mathrm{~Hz}, 1 \mathrm{H}), 8.53 \sim 8.41(\mathrm{~d}$, $J=4 \mathrm{~Hz}, 1 \mathrm{H}), 8.37 \sim 8.34(\mathrm{~m}, 1 \mathrm{H}), 7.62(\mathrm{~s}, 1 \mathrm{H}), 7.45 \sim$ $7.41(\mathrm{~m}, 1 \mathrm{H}), 7.16 \sim 7.14(\mathrm{~d}, J=8.0 \mathrm{~Hz}, 1 \mathrm{H}), 7.07 \sim 6.86$ (m, $2 \mathrm{H}), 6.44 \sim 6.41(\mathrm{~m}, 1 \mathrm{H}), 2.29 \sim 2.19(\mathrm{~s}, 3 \mathrm{H}) ; \mathrm{IR}(\mathrm{KBr})$ $v: 3442.6,3309.0,3207.4,1587.9,1567.9,1528.0,1443.3$, $1403.1,1194.8,793.3 \mathrm{~cm}^{-1}$.

\subsection{5 伊马替尼氨基酸衍生物 $7 \mathbf{a} \sim 7 \mathbf{n}$ 的合成}

向 $100 \mathrm{~mL}$ 四颈瓶中加入二氯甲烷 $(50 \mathrm{~mL})$, 冰浴降 至 $0{ }^{\circ} \mathrm{C}$ 后, 依次加入 $N$-(2-甲基-5-氨基苯基)-4-(3-吡啶 基)嘧啶-2-胺 $(2.20 \mathrm{~g}, 0.008 \mathrm{~mol})$ 和 $(L)-N$-酰化氨基酸 $(0.01 \mathrm{~mol})$, 搅拌 $10 \mathrm{~min}$ 后加入 $O$-苯并三唑- $N, N, N^{\prime}, N^{\prime}$ 四甲基胀四氟硼酸酯(TBTU, $3.60 \mathrm{~g}, 0.012 \mathrm{~mol}$ )及 $N, N$ 二异丙基乙胺(DIEA, $1.40 \mathrm{~g}, 0.012 \mathrm{~mol}$ ), 固体全部溶 解, 控温 $0{ }^{\circ} \mathrm{C}$ 摚拌 $30 \mathrm{~min}$, 撤去冰浴, 逐渐升至室温反 应, 有固体逐渐析出, TLC 跟踪至反应完成[展开剂: $V$ (石油醚)： $V$ (乙酸乙酯 $)=1: 2 \sim 1: 5]$. 抽滤, 滤饼用 $20 \mathrm{~mL}$ 饱和 $\mathrm{Na}_{2} \mathrm{CO}_{3}$ 溶液洗涤, 固体用甲醇/乙酸乙酯 $(V: V=1: 3 \sim 1: 5)$ 重结晶, 得到伊马替尼氨基酸衍生 物 7a $\sim 7 \mathbf{n}$.

[(2S)-2-乙酰胺基]- $N$ - \{4-甲基-3-[[4-(3-吡啶)-2-嘧啶] 氨基]苯基 $\}$ 苯丙酰胺 $(7 \mathbf{a})$ : 淡黄色晶体, 产率 $79.6 \%$. 
m.p. $267.5 \sim 268{ }^{\circ} \mathrm{C},[\alpha]_{\mathrm{D}}^{25}-34.3\left(\right.$ c $\left.1, \mathrm{CH}_{3} \mathrm{OH}\right) ;{ }^{1} \mathrm{H}$ NMR (400 MHz, DMSO- $\left.d_{6}\right) \delta$ : $10.04(\mathrm{~s}, 1 \mathrm{H}), 9.27(\mathrm{~s}, 1 \mathrm{H})$, $8.93(\mathrm{~s}, 1 \mathrm{H}), 8.74 \sim 8.66(\mathrm{~m}, 1 \mathrm{H}), 8.49$ (dd, $J=22.4,6.6$ $\mathrm{Hz}, 2 \mathrm{H}), 8.25(\mathrm{~d}, J=8.2 \mathrm{~Hz}, 1 \mathrm{H}), 7.92(\mathrm{~d}, J=1.9 \mathrm{~Hz}, 1 \mathrm{H})$, $7.53(\mathrm{dd}, J=8.0,4.8 \mathrm{~Hz}, 1 \mathrm{H}), 7.44(\mathrm{~d}, J=5.2 \mathrm{~Hz}, 1 \mathrm{H})$, $7.35 \sim 7.10(\mathrm{~m}, 7 \mathrm{H}), 4.66(\mathrm{td}, J=9.2,5.3 \mathrm{~Hz}, 1 \mathrm{H}), 3.03$ (dd, $J=13.7,5.0 \mathrm{~Hz}, 1 \mathrm{H}), 2.85(\mathrm{dd}, J=13.7,9.5 \mathrm{~Hz}, 1 \mathrm{H})$, $2.20(\mathrm{~s}, 3 \mathrm{H}), 1.80(\mathrm{~s}, 3 \mathrm{H}),{ }^{13} \mathrm{C}$ NMR $\left(100 \mathrm{MHz}\right.$, DMSO- $d_{6}$ ) $\delta: 170.6,169.7,162.0,161.5,159.9,151.8,148.6,138.3$, $137.3,134.9,132.6,130.5,129.6,128.5,127.6,126.7$, 124.2, 116.5, 116.1, 108.0, 55.3, 38.2, 22.8, 18.0; IR (KBr) $v: 3703,3450,3289,1645,1532,1454,1396,798,701$, $566 \mathrm{~cm}^{-1}$; HRMS (ESI-MS) calcd for $\mathrm{C}_{27} \mathrm{H}_{27} \mathrm{~N}_{6} \mathrm{O}_{2}[\mathrm{M}+$ $\mathrm{H}]^{+}$467.2190, found 467.2190.

[(2S)-2-丙酰胺基]- $N$ - $\{4$-甲基-3-[[4-(3-吡啶)-2-嘧啶] 氨基]苯基 $\}$ 苯丙酰胺 (7b): 白色粉末, 产率 77.1\%. m.p. $220.5 \sim 222{ }^{\circ} \mathrm{C} ;[\alpha]_{\mathrm{D}}^{25}-35.6\left(c 0.5, \mathrm{CH}_{3} \mathrm{OH}\right) ;{ }^{1} \mathrm{H} \mathrm{NMR}$ (400 MHz, DMSO- $\left.d_{6}\right) \delta: 10.03(\mathrm{~s}, 1 \mathrm{H}), 9.26(\mathrm{~d}, J=1.7 \mathrm{~Hz}$, $1 \mathrm{H}), 8.92(\mathrm{~s}, 1 \mathrm{H}), 8.69(\mathrm{dd}, J=4.8,1.6 \mathrm{~Hz}, 1 \mathrm{H}), 8.48(\mathrm{dd}$, $J=20.6,6.6 \mathrm{~Hz}, 2 \mathrm{H}), 8.13(\mathrm{~d}, J=8.3 \mathrm{~Hz}, 1 \mathrm{H}), 7.92$ (d, $J=$ $1.8 \mathrm{~Hz}, 1 \mathrm{H}), 7.53(\mathrm{dd}, J=7.9,4.8 \mathrm{~Hz}, 1 \mathrm{H}), 7.43$ (d, $J=5.2$ $\mathrm{Hz}, 1 \mathrm{H}), 7.34 \sim 7.11(\mathrm{~m}, 7 \mathrm{H}), 4.76 \sim 4.58(\mathrm{~m}, 1 \mathrm{H}), 3.14 \sim$ $2.76(\mathrm{~m}, 2 \mathrm{H}), 2.20(\mathrm{~s}, 3 \mathrm{H}), 2.13 \sim 1.98(\mathrm{~m}, 2 \mathrm{H}), 0.90(\mathrm{t}$, $J=7.6 \mathrm{~Hz}, 3 \mathrm{H}) ;{ }^{13} \mathrm{C}$ NMR $\left(100 \mathrm{MHz}\right.$, DMSO- $\left.d_{6}\right) \delta: 173.4$, $170.6,162.0,161.5,159.9,151.84,148.6,138.3,137.3$, $134.8,132.6,130.5,129.6,128.5,127.6,126.7,124.2$, 116.6, 116.1, 108.0, 55.1, 38.3, 28.6, 18.0, 10.1; IR (KBr) $v: 3699,3289,2972,1670,1643,1583,1528,1454,1402$, 797, $697 \mathrm{~cm}^{-1}$; HRMS (ESI-MS) calcd for $\mathrm{C}_{28} \mathrm{H}_{29} \mathrm{~N}_{6} \mathrm{O}_{2}$ $[\mathrm{M}+\mathrm{H}]^{+}$481.2347, found 481.2347.

[(2S)-2-叔丁氧羰基胺基] $-N$ - $\{4$ - 甲基-3-[[4-(3-吡 啶)-2-嘧啶] 氨基] 苯基\} 苯丙酰胺(7c)：白色粉末, 产率 76.9\%. m.p. $205.7 \sim 206.9{ }^{\circ} \mathrm{C} ;[\alpha]_{\mathrm{D}}^{25}-33.5$ (c 1, $\left.\mathrm{CH}_{3} \mathrm{OH}\right) ;{ }^{1} \mathrm{H}$ NMR (400 MHz, DMSO- $\left.d_{6}\right) \delta: 9.97(\mathrm{~s}, 1 \mathrm{H})$, $9.27(\mathrm{~d}, J=1.8 \mathrm{~Hz}, 1 \mathrm{H}), 8.94(\mathrm{~s}, 1 \mathrm{H}), 8.70(\mathrm{~d}, J=4.7 \mathrm{~Hz}$, $1 \mathrm{H}), 8.50(\mathrm{dd}, J=15.8,6.6 \mathrm{~Hz}, 2 \mathrm{H}), 7.93(\mathrm{~s}, 1 \mathrm{H}), 7.61 \sim$ $7.00(\mathrm{~m}, 10 \mathrm{H}), 4.34(\mathrm{~s}, 1 \mathrm{H}), 3.09 \sim 2.74(\mathrm{~m}, 2 \mathrm{H}), 2.21(\mathrm{~s}$, $3 \mathrm{H}), 1.28(\mathrm{~d}, J=31.2 \mathrm{~Hz}, 9 \mathrm{H}) ;{ }^{13} \mathrm{C}$ NMR $(100 \mathrm{MHz}$, DMSO- $\left.d_{6}\right) \delta: 171.0,162.0,161.5,159.9,155.8,151.8$, $148.6,138.4,137.3,134.9,132.6,130.6,129.7,128.4$, $127.5,126.7,124.2,116.4,116.0,108.0,78.5,57.0,38.0$, 28.6, 18.0; IR (KBr) v: 3693, 3337, 2980, 1698, 1573, 1452, 1410, 1163, 1029, 707, $605 \mathrm{~cm}^{-1}$; HRMS (ESI-MS) calcd for $\mathrm{C}_{30} \mathrm{H}_{33} \mathrm{~N}_{6} \mathrm{O}_{3}[\mathrm{M}+\mathrm{H}]^{+}$525.2609, found 525.2606 .

[2-乙酰胺基]- $N$ - $\{4$ - 甲基-3-[[4-(3-吡啶)-2-嘧啶]氨
基]苯基 $\}$ 甘氨酰胺(7d): 白色晶体, 产率 79.8\%. m.p. 212 213.5 ${ }^{\circ} \mathrm{C} ;{ }^{1} \mathrm{H}$ NMR (400 MHz, DMSO- $\left.d_{6}\right) \delta: 9.88$ (s, $1 \mathrm{H}), 9.26(\mathrm{~d}, J=1.9 \mathrm{~Hz}, 1 \mathrm{H}), 8.92(\mathrm{~s}, 1 \mathrm{H}), 8.70(\mathrm{dd}$, $J=4.8,1.5 \mathrm{~Hz}, 1 \mathrm{H}), 8.47$ (ddd, $J=9.8,8.0,3.5 \mathrm{~Hz}, 2 \mathrm{H}$ ), $8.18(\mathrm{t}, J=5.7 \mathrm{~Hz}, 1 \mathrm{H}), 7.88(\mathrm{~d}, J=1.6 \mathrm{~Hz}, 1 \mathrm{H}), 7.54(\mathrm{dd}$, $J=8.0,4.8 \mathrm{~Hz}, 1 \mathrm{H}), 7.43(\mathrm{~d}, J=5.2 \mathrm{~Hz}, 1 \mathrm{H}), 7.30(\mathrm{dd}, J=$ $8.2,1.9 \mathrm{~Hz}, 1 \mathrm{H}), 7.16(\mathrm{~d}, J=8.3 \mathrm{~Hz}, 1 \mathrm{H}), 3.86(\mathrm{~d}, J=5.8$ $\mathrm{Hz}, 2 \mathrm{H}), 2.20$ (s, 3H), 1.89 (s, 3H); ${ }^{13} \mathrm{C}$ NMR $(100 \mathrm{MHz}$, DMSO- $\left.d_{6}\right) \delta: 170.1,168.1,162.0,161.5,159.9,151.8$, $148.6,138.3,137.3,134.9,132.6,130.6,127.4,124.3$, $116.4,115.9,108.0,43.2,22.9,18.0$; IR (KBr) $v: 3433$, 3196, 2905, 1698, 1655, 1557, 1452, 1239, 1311, 1023, $790,705,589 \mathrm{~cm}^{-1}$; HRMS (ESI-MS) calcd for $\mathrm{C}_{20} \mathrm{H}_{21}$ $\mathrm{N}_{6} \mathrm{O}_{2}[\mathrm{M}+\mathrm{H}]^{+}$377.1721, found 377.1717 .

[(2S)-2-乙酰胺基]- $N$ - $\{4$-甲基-3-[[4-(3-吡啶)-2-嘧啶] 氨基]苯基\} 亮酰胺(7e): 淡黄色粉末, 产率 84.7\%. m.p. $207.5 \sim 209{ }^{\circ} \mathrm{C} ;[\alpha]_{\mathrm{D}}^{25}-35.7$ (c 0.8, $\left.\mathrm{CH}_{3} \mathrm{OH}\right) ;{ }^{1} \mathrm{H}$ NMR (400 MHz, DMSO- $\left.d_{6}\right) \delta: 10.01(\mathrm{~s}, 1 \mathrm{H}), 9.26(\mathrm{~s}, 1 \mathrm{H}), 8.94$ (s, 1H), $8.70(\mathrm{~d}, J=4.2 \mathrm{~Hz}, 1 \mathrm{H}), 8.48(\mathrm{dd}, J=22.1,6.5 \mathrm{~Hz}$, 2H), $8.11(\mathrm{~d}, J=7.9 \mathrm{~Hz}, 1 \mathrm{H}), 7.94(\mathrm{~s}, 1 \mathrm{H}), 7.52$ (dd, $J=$ 7.7, $5.0 \mathrm{~Hz}, 1 \mathrm{H}), 7.43(\mathrm{~d}, J=5.1 \mathrm{~Hz}, 1 \mathrm{H}), 7.32$ (d, $J=8.0$ $\mathrm{Hz}, 1 \mathrm{H}), 7.15$ (d, $J=8.2 \mathrm{~Hz}, 1 \mathrm{H}), 4.45$ (dd, $J=14.8,8.1$ $\mathrm{Hz}, 1 \mathrm{H}), 2.19$ (s, 3H), $1.86(\mathrm{~s}, 3 \mathrm{H}), 1.72 \sim 1.41(\mathrm{~m}, 3 \mathrm{H})$, 0.90 (dd, $J=16.7,6.4 \mathrm{~Hz}, 6 \mathrm{H}) ;{ }^{13} \mathrm{C}$ NMR $(100 \mathrm{MHz}$, DMSO- $\left.d_{6}\right) \delta$ : $171.6,169.7,162.0,161.5,159.9,151.8$, $148.6,138.2,137.4,134.8,132.6,130.5,127.5,124.2$, $116.5,116.1,108.0,52.3,41.4,24.8,23.4,22.8,22.2,18.0$; IR (KBr) v: 3445, 3249, 2924, 1649, 1633, 1417, 1299, 891, 804, 633, $483 \mathrm{~cm}^{-1}$; HRMS (ESI-MS) calcd for $\mathrm{C}_{24} \mathrm{H}_{29} \mathrm{~N}_{6} \mathrm{O}_{2}[\mathrm{M}+\mathrm{H}]^{+}$433.2347, found 433.2345.

[(2S)-2-苯甲酰胺基]- $N$ - $\{4$-甲基-3-[[4-(3-吡啶)-2-嘧 啶]氨基]苯基\}苯丙酰胺(7f): 白色粉末，产率 76.2\%. m.p. $258 \sim 259.5{ }^{\circ} \mathrm{C} ;[\alpha]_{\mathrm{D}}^{25}-34.5\left(c \quad 1, \mathrm{CH}_{3} \mathrm{OH}\right) ;{ }^{1} \mathrm{H}$ NMR (400 MHz, DMSO- $\left.d_{6}\right) \delta: 10.18(\mathrm{~s}, 1 \mathrm{H}), 9.27$ (d, $J=$ $1.9 \mathrm{~Hz}, 1 \mathrm{H}), 8.94(\mathrm{~s}, 1 \mathrm{H}), 8.81 \sim 8.60(\mathrm{~m}, 2 \mathrm{H}), 8.57 \sim 8.27$ (m, 2H), $7.99(\mathrm{~d}, J=1.9 \mathrm{~Hz}, 1 \mathrm{H}), 7.93 \sim 7.76(\mathrm{~m}, 2 \mathrm{H})$, $7.60 \sim 7.38(\mathrm{~m}, 7 \mathrm{H}), 7.36 \sim 7.23(\mathrm{~m}, 3 \mathrm{H}), 7.23 \sim 7.11(\mathrm{~m}$, $2 \mathrm{H}), 4.87(\mathrm{td}, J=9.6,5.0 \mathrm{~Hz}, 1 \mathrm{H}), 3.26 \sim 2.97(\mathrm{~m}, 2 \mathrm{H})$, $2.22(\mathrm{~s}, 3 \mathrm{H}) ;{ }^{13} \mathrm{C}$ NMR $\left(100 \mathrm{MHz}, \mathrm{DMSO}-d_{6}\right) \delta: 170.7$, $166.9,162.0,161.5,159.9,151.8,148.6,138.6,138.3$, $137.3,134.9,134.4,132.6,131.8,130.6,129.7,128.6$, $127.9,127.5,126.7,124.2,116.5,116.0,108.0,56.3,37.7$, 18.0; IR (KBr) v: 3432, 3295, 2924, 1668, 1637, 1584, 1529, 1399, 801, 698, $567 \mathrm{~cm}^{-1}$; HRMS (ESI-MS) calcd for $\mathrm{C}_{32} \mathrm{H}_{29} \mathrm{~N}_{6} \mathrm{O}_{2}[\mathrm{M}+\mathrm{H}]^{+} 529.2347$, found 529.2337.

[(2S)-2-苯乙酰胺基]- $N$ - $\{4$-甲基-3-[[4-(3-吡啶)-2-嘧 
啶]氨基]苯基\} 苯丙酰胺 $\mathbf{7 g}$ ): 淡黄色粉末, 产率 $87.1 \%$. m.p. $234.5 \sim 235{ }^{\circ} \mathrm{C} ;[\alpha]_{\mathrm{D}}^{25}-33.8$ (c 1, $\left.\mathrm{CH}_{3} \mathrm{OH}\right) ;{ }^{1} \mathrm{H}$ NMR (400 MHz, DMSO- $\left.d_{6}\right) \delta: 10.07$ (s, 1H), $9.27(\mathrm{~s}, 1 \mathrm{H})$, $8.93(\mathrm{~s}, 1 \mathrm{H}), 8.69$ (d, $J=4.8 \mathrm{~Hz}, 1 \mathrm{H}), 8.58 \sim 8.39(\mathrm{~m}, 3 \mathrm{H})$, $7.91(\mathrm{~s}, 1 \mathrm{H}), 7.58 \sim 7.41(\mathrm{~m}, 2 \mathrm{H}), 7.35 \sim 7.04(\mathrm{~m}, 12 \mathrm{H})$, $4.69(\mathrm{td}, J=9.2,5.1 \mathrm{~Hz}, 1 \mathrm{H}), 3.57 \sim 3.34(\mathrm{~m}, 2 \mathrm{H}), 2.20(\mathrm{~s}$, 2H), $1.24(\mathrm{~s}, 3 \mathrm{H}) ;{ }^{13} \mathrm{C}$ NMR (100 MHz, DMSO-d $d_{6} \delta$ : $170.5,162.0,161.5,159.9,151.7,148.6,138.3,138.0$, $137.2,136.7,134.9,132.7,130.6,129.7,129.4,128.5$, $127.6,126.7,124.3,116.5,116.1,108.0,55.3,42.3,38.4$, 18.0; IR (KBr) v: 3429, 3297, 3025, 1630, 1581, 1454, 1394, 1084, 796, 701, $572 \mathrm{~cm}^{-1}$; HRMS (ESI-MS) calcd for $\mathrm{C}_{33} \mathrm{H}_{31} \mathrm{~N}_{6} \mathrm{O}_{2} \quad[\mathrm{M}+\mathrm{H}]^{+}$543.2503, found 543.2505.

[(2S)-2-对甲苯甲酰胺基] $-N$ - $\{4$ - 甲基-3-[[4-(3-吡 啶)-2-嘧啶]氨基]苯基 $\}$ 苯丙酰胺(7h): 淡黄色粉末，产 率 75.5\%. m.p. $235 \sim 236.3{ }^{\circ} \mathrm{C} ;[\alpha]_{\mathrm{D}}^{25}-33.3$ (c 1, $\left.\mathrm{CH}_{3} \mathrm{OH}\right) ;{ }^{1} \mathrm{H}$ NMR $\left(400 \mathrm{MHz}, \mathrm{DMSO}-d_{6}\right) \delta: 10.18(\mathrm{~s}, 1 \mathrm{H})$, $9.27(\mathrm{~d}, J=1.8 \mathrm{~Hz}, 1 \mathrm{H}), 8.94(\mathrm{~s}, 1 \mathrm{H}), 8.70 \sim 8.59$ (m, $2 \mathrm{H})$, 8.48 (dd, $J=10.2,8.0 \mathrm{~Hz}, 2 \mathrm{H}), 7.99$ (s, 1H), 7.75 (d, $J=$ $8.2 \mathrm{~Hz}, 2 \mathrm{H}), 7.54 \sim 7.38(\mathrm{~m}, 4 \mathrm{H}), 7.36 \sim 7.13(\mathrm{~m}, 7 \mathrm{H}), 4.86$ (dd, $J=8.9,5.4 \mathrm{~Hz}, 1 \mathrm{H}), 3.24 \sim 3.04(\mathrm{~m}, 2 \mathrm{H}), 2.35(\mathrm{~s}, 3 \mathrm{H})$, $2.22(\mathrm{~s}, 3 \mathrm{H}) ;{ }^{13} \mathrm{C}$ NMR (100 MHz, DMSO- $\left.d_{6}\right) \delta: 170.8$, $166.8,162.0,161.5,159.9,151.8,148.6,141.7,138.7$, 138.3 , 137.4, 134.9, 132.6, 131.6, 130.6, 129.7, 129.1, $128.5,127.9,124.2,116.4,116.0,107.9,56.2,37.7,21.4$, 18.0; IR (KBr) v: 3448, 3285, 3031, 1670, 1632, 1530, 1504, 885, 704, 666, $572 \mathrm{~cm}^{-1}$; HRMS (ESI-MS) calcd for $\mathrm{C}_{33} \mathrm{H}_{31} \mathrm{~N}_{6} \mathrm{O}_{2}[\mathrm{M}+\mathrm{H}]^{+}$543.2503, found 543.2501.

[2-苯乙酰胺基]- $N$-\{4-甲基-3-[[4-(3-吡啶)-2-嘧啶]氨 基]苯基 $\}$ 甘氨酰胺 $(7 \mathbf{i})$ : 白色晶体, 产率 78.8\%. m.p. $239 \sim 240.5{ }^{\circ} \mathrm{C}$; ${ }^{1} \mathrm{H}$ NMR (400 MHz, DMSO- $d_{6}$ ) $\delta: 9.91$ (s, 1H), 9.26 (d, J=1.7 Hz, 1H), 8.92 (s, 1H), 8.69 (dd, $J=4.8,1.6 \mathrm{~Hz}, 1 \mathrm{H}), 8.47(\mathrm{dd}, J=8.0,3.5 \mathrm{~Hz}, 2 \mathrm{H}), 8.36(\mathrm{t}$, $J=5.7 \mathrm{~Hz}, 1 \mathrm{H}), 7.88$ (d, $J=1.7 \mathrm{~Hz}, 1 \mathrm{H}), 7.53$ (dd, $J=7.5$, $4.8 \mathrm{~Hz}, 1 \mathrm{H}), 7.43(\mathrm{~d}, J=5.2 \mathrm{~Hz}, 1 \mathrm{H}), 7.35 \sim 7.13(\mathrm{~m}, 7 \mathrm{H})$, $3.91(\mathrm{~d}, J=5.8 \mathrm{~Hz}, 2 \mathrm{H}), 3.52(\mathrm{~s}, 2 \mathrm{H}), 2.20(\mathrm{~s}, 3 \mathrm{H}) ;{ }^{13} \mathrm{C}$ NMR (100 MHz, DMSO- $\left.d_{6}\right) \delta$ : 171.0, 167.9, 162.1, 161.5, $159.9,151.8,148.6,138.3,137.3,136.7,134.9,132.6$, $130.6,129.5,128.6,127.5,126.7,124.3,116.3,115.9$, 108.0, 43.2, 42.5, 18.0; IR (KBr) v: 3267, 3056, 2984, 1697, 1654, 1534, 1409, 1244, 1021, 821, 703, $645 \mathrm{~cm}^{-1}$; HRMS (ESI-MS) calcd for $\mathrm{C}_{26} \mathrm{H}_{25} \mathrm{~N}_{6} \mathrm{O}_{2}[\mathrm{M}+\mathrm{H}]{ }^{+}$ 453.2034, found 453.2026.

[(2S)-2-苯甲酰胺基]- $N$-\{4-甲基-3-[[4-(3-吡啶)-2-嘧 啶]氨基]苯基 $\}$ 丙酰胺 $(7 \mathbf{j})$ : 淡黄色粉末，产率 76.1\%. m.p. $206.0 \sim 207.5{ }^{\circ} \mathrm{C} ;[\alpha]_{\mathrm{D}}^{25}+13.8\left(c 1, \mathrm{CH}_{3} \mathrm{OH}\right) ;{ }^{1} \mathrm{H}$
NMR (400 MHz, DMSO- $\left.d_{6}\right) \delta: 10.02(\mathrm{~s}, 1 \mathrm{H}), 9.26(\mathrm{~s}, 1 \mathrm{H})$, 8.94 (s, 1H), 8.64 (dd, $J=14.6,5.5 \mathrm{~Hz}, 2 \mathrm{H}), 8.48$ (dd, $J=$ $19.4,6.4 \mathrm{~Hz}, 2 \mathrm{H}), 8.02 \sim 7.87(\mathrm{~m}, 3 \mathrm{H}), 7.59 \sim 7.39(\mathrm{~m}$, $5 \mathrm{H}), 7.31(\mathrm{~d}, J=8.0 \mathrm{~Hz}, 1 \mathrm{H}), 7.16(\mathrm{~d}, J=8.3 \mathrm{~Hz}, 1 \mathrm{H})$, $4.73 \sim 4.54(\mathrm{~m}, 1 \mathrm{H}), 2.20(\mathrm{~s}, 3 \mathrm{H}), 1.45$ (d, J=7.1 Hz, 3H); ${ }^{13} \mathrm{C}$ NMR (100 MHz, DMSO- $\left.d_{6}\right) \delta: 171.7,166.7,162.0$, $161.5,159.9,151.7,148.6,138.3,137.5,134.9,134.4$, $132.6,131.7,130.5,128.6,128.0,127.3,124.2,116.4$, 115.9, 108.0, 50.3, 18.3, 18.0; IR (KBr) v: 3440, 3302, 2983, 1668, 1634, 1582, 1529, 1397, 1010, 792, 662, 619 $\mathrm{cm}^{-1}$; HRMS (ESI-MS) calcd for $\mathrm{C}_{26} \mathrm{H}_{25} \mathrm{~N}_{6} \mathrm{O}_{2}[\mathrm{M}+\mathrm{H}]^{+}$ 453.2034, found 453.2034.

[(2S)-2-苯乙酰胺基]- $N$-\{甲基-3-[[4-(3-吡啶)-2-嘧 啶]氨基]苯基\}丙酰胺 $(7 \mathbf{k})$ : 淡黄色粉末，产率 75.0\%. m.p. $237 \sim 238.3{ }^{\circ} \mathrm{C} ;[\alpha]_{\mathrm{D}}^{25}+13.6$ (c 1, $\left.\mathrm{CH}_{3} \mathrm{OH}\right) ;{ }^{1} \mathrm{H}$ NMR (400 MHz, DMSO-d $)) \delta: 9.96(\mathrm{~s}, 1 \mathrm{H}), 9.26$ (d, $J=$ $1.7 \mathrm{~Hz}, 1 \mathrm{H}), 8.94$ (s, 1H), 8.69 (dd, $J=4.8,1.6 \mathrm{~Hz}, 1 \mathrm{H})$, $8.51(\mathrm{~d}, J=5.1 \mathrm{~Hz}, 1 \mathrm{H}), 8.48 \sim 8.43(\mathrm{~m}, 1 \mathrm{H}), 8.39(\mathrm{~d}$, $J=7.3 \mathrm{~Hz}, 1 \mathrm{H}), 7.92(\mathrm{~d}, J=1.8 \mathrm{~Hz}, 1 \mathrm{H}), 7.51$ (dd, $J=7.9$, $4.9 \mathrm{~Hz}, 1 \mathrm{H}), 7.43$ (d, $J=5.2 \mathrm{~Hz}, 1 \mathrm{H}), 7.27$ (d, $J=4.4 \mathrm{~Hz}$, $5 \mathrm{H}), 7.23 \sim 7.13(\mathrm{~m}, 2 \mathrm{H}), 4.43$ (p, $J=7.0 \mathrm{~Hz}, 1 \mathrm{H}), 3.49$ (s, 2H), 2.19 (s, 3H), 1.31 (d, $J=4.0 \mathrm{~Hz}, 3 \mathrm{H}) ;{ }^{13} \mathrm{C}$ NMR (100 $\left.\mathrm{MHz}, \mathrm{DMSO}-d_{6}\right) \delta: 171.5,70.4,162.0,161.5,159.9$, $151.8,148.6,138.3,137.4,136.8,134.8,132.6,130.6$, $129.5,128.6,127.5,126.7,124.2,116.4,115.9,108.0$, $49.5,42.3,18.8,18.0$; IR (KBr) $v: 3440,3303,2983,1668$, 1634, 1582, 1529, 1387, 1023, 791, 691, 662, $586 \mathrm{~cm}^{-1}$; HRMS (ESI-MS) calcd for $\mathrm{C}_{27} \mathrm{H}_{27} \mathrm{~N}_{6} \mathrm{O}_{2} \quad[\mathrm{M}+\mathrm{H}]$ 467.2190, found 467.2183.

[(2S)-2-对甲苯甲酰胺基]- $N$-\{4-甲基-3-[[4-(3-吡 啶)-2-嘧啶]氨基]苯基 $\}$ 丙酰胺(71): 淡黄色粉末，产率 $70 \%$. m.p. $240 \sim 241.5{ }^{\circ} \mathrm{C}$; $[\alpha]_{\mathrm{D}}^{25}+14.2$ (c 1, $\left.\mathrm{CH}_{3} \mathrm{OH}\right) ;{ }^{1} \mathrm{H}$ NMR (400 MHz, DMSO- $\left.d_{6}\right) \delta: 10.01(\mathrm{~s}, 1 \mathrm{H}), 9.26(\mathrm{~s}, 1 \mathrm{H})$, $8.94(\mathrm{~s}, 1 \mathrm{H}), 8.66(\mathrm{~d}, J=4.5 \mathrm{~Hz}, 1 \mathrm{H}), 8.52$ (t, $J=6.8 \mathrm{~Hz}$, 2H), 8.46 (d, $J=8.0 \mathrm{~Hz}, 1 \mathrm{H}), 7.98$ (s, 1H), 7.84 (d, $J=8.0$ $\mathrm{Hz}, 2 \mathrm{H}), 7.49$ (dd, $J=7.8,4.8 \mathrm{~Hz}, 1 \mathrm{H}), 7.42$ (d, $J=5.1$ $\mathrm{Hz}, 1 \mathrm{H}), 7.29$ (dd, $J=14.1,8.2 \mathrm{~Hz}, 3 \mathrm{H}), 7.16$ (d, $J=8.3$ $\mathrm{Hz}, 1 \mathrm{H}), 4.68 \sim 4.55(\mathrm{~m}, 1 \mathrm{H}), 2.36(\mathrm{~s}, 3 \mathrm{H}), 2.20(\mathrm{~s}, 3 \mathrm{H})$, $1.44(\mathrm{~d}, J=7.1 \mathrm{~Hz}, 3 \mathrm{H}) ;{ }^{13} \mathrm{C}$ NMR $\left(100 \mathrm{MHz}, \mathrm{DMSO}-d_{6}\right)$ $\delta: 171.8,166.6,162.0,161.5,159.9,151.7,148.6,141.6$, $138.3,137.5,134.9,132.6,131.6,130.5,129.1,128.0$, $127.3,124.2,116.4,115.9,108.0,50.3,21.4,18.3,18.0$; IR (KBr) v: 3407, 3286, 3203, 2922, 1691, 1633, 1571, 1490, 1074, 971, 882, 757, $582 \mathrm{~cm}^{-1}$; HRMS (ESI-MS) calcd for $\mathrm{C}_{27} \mathrm{H}_{27} \mathrm{~N}_{6} \mathrm{O}_{2} \quad[\mathrm{M}+\mathrm{H}]^{+}$467.2190, found 467.2188.

[(2S)-2-苯乙酰胺基]- $N$-\{4-甲基-3-[[4-(3-吡啶)-2-嘧 
啶]氨基]苯基\} 缬氨酰胺(7m): 淡黄色粉末, 产率 75.7\%. m.p. $223.5 \sim 224{ }^{\circ} \mathrm{C}$; $[\alpha]_{\mathrm{D}}^{25}+27.6$ (c 0.6, $\left.\mathrm{CH}_{3} \mathrm{OH}\right) ;{ }^{1} \mathrm{H}$ NMR (400 MHz, DMSO- $\left.d_{6}\right) \delta: 10.04(\mathrm{~s}, 1 \mathrm{H}), 9.26(\mathrm{~s}, 1 \mathrm{H})$, $8.94(\mathrm{~s}, 1 \mathrm{H}), 8.69$ (d, $J=3.8 \mathrm{~Hz}, 1 \mathrm{H}), 8.48$ (dd, $J=24.4$, $6.5 \mathrm{~Hz}, 2 \mathrm{H}), 8.26(\mathrm{~d}, J=8.7 \mathrm{~Hz}, 1 \mathrm{H}), 7.94(\mathrm{~s}, 1 \mathrm{H}), 7.53 \sim$ $7.39(\mathrm{~m}, 2 \mathrm{H}), 7.28(\mathrm{~d}, J=4.2 \mathrm{~Hz}, 5 \mathrm{H}), 7.22 \sim 7.12(\mathrm{~m}$, 2H), $4.30(\mathrm{t}, J=8.0 \mathrm{~Hz}, 1 \mathrm{H}), 3.54(\mathrm{dd}, J=36.9,13.8 \mathrm{~Hz}$, 2H), 2.19 (s, 3H), $2.02 \quad(\mathrm{dd}, J=12.0,4.0 \mathrm{~Hz}, 1 \mathrm{H}), 0.89$ (d, $J=5.9 \mathrm{~Hz}, 6 \mathrm{H}) ;{ }^{13} \mathrm{C}$ NMR $\left(100 \mathrm{MHz}, \mathrm{DMSO}-d_{6}\right) \delta: 170.7$, $170.5,162.0,161.5,159.9,151.8,148.6,138.3,137.1$, $134.8,132.6,130.6,129.4,128.6,127.5,126.7,124.2$, $116.4,116.0,108.0,59.0,42.4,31.3,19.6,18.9$, 18.0; IR (KBr) v: 3450, 3251, 2970, 2926, 1677, 1642, 1604, 1583, 1444, 1236, 789, 709, $621 \mathrm{~cm}^{-1}$; HRMS (ESI-MS) calcd for $\mathrm{C}_{29} \mathrm{H}_{31} \mathrm{~N}_{6} \mathrm{O}_{2}[\mathrm{M}+\mathrm{H}]^{+}$495.2503, found 495.2494

[2-对甲苯甲酰胺基]- $N$-\{4-甲基-3-[[4-(3-吡啶)-2-嘧 啶]氨基]苯基 $\}$ 甘氨酰胺 (7n): 白色晶体, 产率 $73.8 \%$. m.p. $245.5 \sim 247{ }^{\circ} \mathrm{C} ;{ }^{1} \mathrm{H}$ NMR (400 MHz, DMSO-d $d_{6} \delta$ : $10.00(\mathrm{~s}, 1 \mathrm{H}), 9.26(\mathrm{~s}, 1 \mathrm{H}), 8.92(\mathrm{~s}, 1 \mathrm{H}), 8.71(\mathrm{~d}, J=29.7$ $\mathrm{Hz}, 2 \mathrm{H}), 8.50(\mathrm{~d}, J=12.5 \mathrm{~Hz}, 2 \mathrm{H}), 8.14 \sim 7.74(\mathrm{~m}, 3 \mathrm{H})$, 7.35 (dd, $J=89.7,43.5 \mathrm{~Hz}, 6 \mathrm{H}), 4.07$ (s, 2H), 2.29 (d, $J=$ $60.4 \mathrm{~Hz}, 6 \mathrm{H}) ;{ }^{13} \mathrm{C}$ NMR $\left(100 \mathrm{MHz}, \mathrm{DMSO}-d_{6}\right) \delta: 168.1$, $167.0,162.1,161.5,159.8,151.7,148.5,141.7,138.3$, $137.4,134.9,132.6,131.7,130.6,129.3,127.8,127.3$, 124.3, 116.3, 115.9, 108.0, 43.7, 21.4, 18.0; IR (KBr) $v$ : 3349, 3279, 3050, 2921, 1706, 1637, 1529, 1494, 1426, 1229, 878, 748, $587 \mathrm{~cm}^{-1}$; HRMS (ESI-MS) calcd for $\mathrm{C}_{26} \mathrm{H}_{25} \mathrm{~N}_{6} \mathrm{O}_{2}[\mathrm{M}+\mathrm{H}]^{+}$453.2034, found 453.2033.

\section{3 抗肿瘤细胞毒活性实验}

对合成的 14 个化合物 7a $7 \mathbf{n}$ 进行了体外细胞毒活 性测试. 采用四甲基偶氮唑盐(MTT)微量酶反应比色法 测定其对人白血病细胞(K562)、人肺癌细胞(A549)、肝 癌细胞(HepG-2)体外抑制作用. 取处于对数生长期的状 态良好的细胞一瓶, 加入 $0.25 \mathrm{wt} \%$ 胰蛋白酶消化液消 化, 使贴壁细胞脱落, 计数, 制成 $2 \times 10^{4} \sim 4 \times 10^{4}$ 个细 胞 $/ \mathrm{mL}$ 的悬液. 接种于 96 孔板上, $180 \mu \mathrm{L} /$ 孔, 置恒温 $\mathrm{CO}_{2}$ 培养箱中培养 $24 \mathrm{~h}$, 换液, 加入受试化合物的 DMSO 溶液, $20 \mu \mathrm{L} /$ 孔, 再加入含体积分数为 $10 \%$ 的血 清培养液 $80 \mu \mathrm{L}$, 培养 $48 \mathrm{~h}$, 将 MTT 加入 96 孔板中, 20 $\mu \mathrm{L} /$ 孔，培养箱中反应 $4 \mathrm{~h}$. 吸去上清液，加入 DMSO, $150 \mu \mathrm{L} /$ 孔, 平板摇床上振摇 $5 \mathrm{~min}$. 用酶联免疫检测仪 在波长为 $540 \mathrm{~nm}$ 处测定每孔的吸光值(OD 值), 计算对 细胞增殖的抑制率. 以相应溶媒作阴性对照, 以伊马替 尼为阳性对照. 计算抑制率采用公式: 抑制率 $(\%)=($ 空 白吸光光度值一给药组吸光度值)/空白吸光度值 $\times$ $100 \%$. 目标化合物体外蛋白酪氨酸激酶抑制活性实验 仍在研究中.

辅助材料(Supporting Information) 化合物 7a $\sim 7 \mathrm{n}$ 质 谱、核磁共振氢谱、碳谱和红外图谱. 这些材料可以免 费从本刊网站(http://sioc-journal.cn/)上下载.

\section{References}

[1] Guo, X.-N.; Ding, J. Chin. J. Med. Chem. 2003, 34, 183 (in Chinese). (郭晓宁，丁健，中国新药杂志, 2003, 34, 183.)

[2] Wang, S. H. J. Leuk. Lymphoma 2002, 11, 29 (in Chinese). (张穗慧, 白血病·淋巴瘤, 2002, 11, 29.)

[3] Guilhot, F.; Chastang, C.; Michallet, M.; Guercl, A.; Harousseallu, J. L.; Maloisel, F.; Bouabdallah, R.; Gugotat, D.; Cheron, N.; Nicolini, F.; Abgraii, J. F.; Tanzer, J. N. Engl. J. Med. 1997, 337, $223 .$.

[4] Nowell, P. C.; Hungerford, D. A. Science 1960, 132, 1497.

[5] Chronic Myeloid Leukemia Trialists' Collaborative Group J. Natl. Cancer Inst. 1997, 89, 1616.

[6] O'Brien, S. G.; Guilhot, F.; Larson, R. A. N. Engl. J. Med. 2003, $348,994$.

[7] Gambacorti Passerini, C. B.; Gunby, R. H.; Piazza, R. J. Lancet Oncol. 2003, 4, 75.

[8] Mahon, F. X.; Faberes, C.; Pueyo, S. Blood 1998, 92, 4059.

[9] Zheng, Y.-G.; Chen, D.-J.; Zhu, B.-Q. Chin. J. Med. Chem. 2009, 18, 189 (in Chinese).

(郑玉果，陈代杰，朱宝泉，中国新药杂志，2009, 18, 189.)

[10] Sawyers, C. L.; Hohhasus, A.; Feldman, E.; Goldman, J. M.; Miller, C. B.; Ottmann, O. G.; Schiffer, C. A.; Talpaz, M.; Guilhot, F.; Deininger, M. W. N.; Fischer, T.; OBrien, S. G.; Stone, R. M. Blood 2002, 99, 3530.

[11] Kil, K. E.; Ding, Y. S.; Lin, K. S. Nucl. Med. Biol. 2007, 34, 153.

[12] Kompella, A. K.; Adibnatla, K. S.; Bhujanga, R. WO 2006027795 , 2006 [Chem. Abstr. 2006, 144, 312102].

[13] Buerger, H. M.; Caravatti, G.; Zimmerman, J. WO 2002022597, 2002 [Chem. Abstr. 2002, 136, 2476057].

[14] Jur, G. Z.; Wallba, C. H. US 5521184, 1996 [Chem. Abstr. 1996, $125,114681]$.

[15] Li, M.-D.; Li, D.; Ji, M. Chin. Pharm. J. 2008, 43, 228 (in Chinese).

(李铭东, 李东, 吉民, 中国药学杂志, 2008, 43, 228.) 